\title{
Evaluation of aftershock hazard of the 2018 M 6.2 Hualien earthquake
}

\author{
Yuh-Ing Chen ${ }^{1, *}$, Jann-Yenq Liu ${ }^{2}$, and Zih-Syuan Jian ${ }^{1}$ \\ ${ }^{1}$ Graduate Institute of Statistics, National Central University, Taoyuan City, Taiwan \\ ${ }^{2}$ Graduate Institute of Space Science, National Central University, Taoyuan City, Taiwan
}

\begin{abstract}
Article history:
Received 31 July 2018

Revised 1 December 2018

Accepted 9 December 2018

Keywords:

Bayesian information criterion, Double-sequence aftershock hazard model, Maximum likelihood estimate, Reasenberg-Jones model

Citation:

Chen, Y.-I., J.-Y. Liu, and Z.-S. Jian, 2019: Evaluation of aftershock hazard of the 2018 M 6.2 Hualien earthquake. Terr. Atmos. Ocean. Sci., 30, 411-421, doi: 10.3319/TAO.2018.12.09.01
\end{abstract}

\begin{abstract}
This paper presents a near real-time evaluation of earthquake hazard after the shallow $\mathrm{M}_{\mathrm{L}}$ 6.2 Hualien shock of depth $10.6 \mathrm{~km}$ occurred 2350 LT, 6 February 2018 at epicenter $\left(24.10^{\circ} \mathrm{N}, 121.73^{\circ} \mathrm{E}\right)$. Since a large aftershock intends to bring up a new aftershock sequence, we develop a time-magnitude hazard function for the double aftershock sequences. Note that the double-sequence aftershock hazard model can be regarded as a generalization of the Reasenberg-Jones (RJ) model and hence is denoted by DSRJ. For a near real-time evaluation of the aftershock hazard in the golden window particularly for emergent rescue work, the DSRJ or RJ model is estimated based on early aftershock sequence and then the occurrence rate and number of the forthcoming aftershocks in 3 days after the Hualien main shock are forecasted. Results of a data analysis show that the DSRJ model is better than the RJ model on near real-time assessment of short-term aftershock hazard. This provides an evidence that the aftershocks occurred in a short time after the Hualien main shock may not be a single aftershock sequence.
\end{abstract}

\section{INTRODUCTION}

Moderate to large aftershocks followed by a shallow large main shock may bring a significant hazard to the area with weakened structures. To help decision makers to determine a low-risk emergent rescue work or an optimal treatment of damaged structures, the information about the near real-time hazard of such aftershocks is usually of highly demand. Therefore, it is of great importance to evaluate the hazard of forthcoming aftershocks in a short time after the drastic main shock. In the study of aftershock hazard, the Omori-Utsu law (Omori 1894; Utsu 1961; Utsu et al. 1995) reasonably states the time-decaying occurrence rate of one single aftershock sequence. Moreover, the Gutenberg-Richter law (Gutenberg and Richter 1944) is traditionally used to describe the frequency-magnitude of earthquakes in an area. Therefore, Reasenberg and Jones $(1989,1994)$ combined the G-R and Omori-Utsu laws to obtain the RJ model for describing the occurrence rate of aftershocks with magnitude larger than $m$. Note that the RJ model has been widely applied to evaluate the aftershock hazard in California (Wi-

\footnotetext{
* Corresponding author

E-mail:ychen5325@gmail.com
}

emer et al. 2002; Gerstenberger et al. 2005, 2007) and Japan (Omi et al. 2013).

Nevertheless, the aftershock activity may not be best described by the Omori-Utsu law, especially when aftershocks trigger interactively. Hence, Ogata (1988) proposed the epidemic-type aftershock sequence (ETAS) model in which the aftershock hazard is expressed as a weighted sum of Omori-Utsu models shifted in time. Note that the estimation of the ETAS model is time-consuming. Therefore, to have a fast and efficient near real-time assessment of aftershock hazard, a detailed investigation of the early aftershock sequence along with an easy computed yet appropriate aftershock hazard model are necessary.

Although the Omori-Utsu law or the RJ model can be easily used to evaluate the hazard of one single aftershock sequence, it occurs often that large aftershocks after the main shock may bring up a secondary aftershock sequence and hence the occurrence rate of aftershocks increases dramatically. This feature motivates the development of a double-sequence aftershock hazard model in this paper so that an evaluation of the short-term aftershock hazard after the main shock can be evaluated more efficiently. In the 
proposed aftershock hazard model, the decaying occurrence rate of each sequence follows the Omori-Utsu law and the Gutenberg-Richter law describes the magnitude distribution of aftershocks. Hence, the proposed aftershock hazard model can be regarded as a generalization of the RJ model to the double aftershock sequences.

Note that the $\mathrm{M}_{\mathrm{L}} 6.2$ Hualien main shock, a shallow earthquake of depth $10.6 \mathrm{~km}$ on 6 February 2018, caused collapse of four buildings and hence many deaths and injuries. After the main shock, felt aftershocks kept occurring while the emergent rescue work was ongoing. A fast and efficient evaluation of aftershock hazard is then particularly needed at that moment. Therefore, in this paper, we implement a near real-time forecast of forthcoming aftershocks based on earthquake data available after the $\mathrm{M}_{\mathrm{L}} 6.2$ Hualien main shock. In the remainder of the paper, the RJ model is reviewed, the double-sequence aftershock model is proposed, and the Bayesian Information Criterion (Schwarz 1978) or BIC for model selection is introduced in section 2. Results of an analysis of earthquakes after the Hualien main shock are presented in section 3. Finally, in section 4 , we give a discussion of strengths and limitations of the model-based evaluation of aftershock hazard together with conclusions for the research work.

\section{MODELS AND METHODOLOGY}

\subsection{Reasenberg-Jones Model}

The frequency-magnitude distribution of earthquakes in the Gutenberg-Richter (Gutenberg and Richter 1944) law is

$\log _{10} N(m)=a-b m, m>M_{c}$

where $N(m)$ is the number of earthquakes with magnitude $M$ greater than $m$, denoted by $M>m, a$ is a constant related to the activity, $b$ measures the ratio of small to large earthquakes and areas of large slip release may correlated with high $b$ value (Wiemer and Katsumata 1999), and $M_{c}$ is the cut-off magnitude for a complete earthquake catalogue. In general, the magnitude of earthquakes is distributed according to an exponential distribution left-truncated at $M_{c}$. The probability of observing an $M>m$ earthquake is then given by

$S(m)=\exp \left[-\beta\left(m-M_{c}\right)\right], m>M_{c}$

where $\beta=b \ln 10$. Note that the maximum likelihood estimate (MLE) of the parameter $b$ and its standard error can be obtained from Aki (1965). Hence, the MLE of $\beta$ along with its standard error can be solved accordingly.

This paper considers the simplified Omori-Utsu law (Utsu et al. 1995) states the time-decaying occurrence rate of a single aftershock sequence as $\lambda_{1}(t)=\frac{e^{\alpha}}{(t+c)^{p}}, t>0$

where $t$ is the elapsed time after the main shock, the constant $\alpha$ reflects the activity of aftershocks under study, $c$ is the time delay designed to avoid the divergence of the rate when $t$ approaches to zero, and the power exponent $p$ measures the decaying rate of aftershocks. Note that the $p$ value may relate to the temperature of the aftershock zone (Kisslinger and Jones 1991). Moreover, the $c$ value reflects the systematic behaviour of early aftershocks (Nanjo et al. 2007) and depends on the faulting style and hence governed by the state of stress (Narteau et al. 2009). Therefore, the number of aftershocks under study occurring in the time range $\left(T_{1}, T_{2}\right), 0<T_{1}<T_{2}$, can be expressed as

$N=\int_{T_{1}}^{T_{2}} \lambda_{1}(t) d t=A\left(T_{1}, T_{2}, c, p\right)$

where

$A\left(T_{1}, T_{2}, c, p\right)=\left\{\begin{array}{cc}\frac{\left[\left(T_{2}+c\right)^{1-p}-\left(T_{1}+c\right)^{1-p}\right]}{(1-p)} & \text { for } p \neq 1 \\ \ln \left(T_{2}+c\right)-\ln \left(T_{1}+c\right) & \text { for } p=1\end{array}\right.$

Basically, the MLEs of the related parameters can be solved by maximizing the likelihood function in Ogata (1983). Therefore, the Omori-Utsu model can be estimated based on available data of aftershocks and the occurrence rate in Eq. (3) or number of aftershocks in Eq. (4) can then be forecasted based on the estimated Omori-Utsu model.

Assuming that the magnitude and occurrence time of aftershocks are independent, the RJ model (Reasenberg and Jones 1989,1994$)$ describes the occurrence rate of $M>m$ aftershocks with magnitude $m$ or lager as

$\lambda_{1}(t, m)=\lambda_{1}(t) S(m), t>0$ and $m>M_{c}$

where $S(m)$ and $\lambda_{1}(t)$ are given in Eqs. (2) and (3), respectively. Hence, the number of $M>m$ aftershocks occurring in the time range $\left(T_{1}, T_{2}\right), 0<T_{1}<T_{2}$, can be evaluated as

$N_{1}(m)=S(m) \int_{T_{1}}^{T_{2}} \lambda_{1}(t) d t=S(m) N$

Note that the MLEs of the parameters involved in the RJ model can be estimated separately in Eqs. (2) and (3). Therefore, the occurrence rate and the number of aftershocks in Eqs. (6) and (7), respectively, can be forecasted accordingly. 


\subsection{Double-Sequence Aftershock Hazard Model}

An aftershock of large magnitude may trigger a secondary aftershock sequence, a generalization of the Omori-Utsu law to the double aftershock sequences is then considered and the occurrence rate of aftershocks can be written as

$\lambda_{2}(t)=\frac{e^{\alpha_{1}}}{(t+c)^{p}}+\frac{e^{\alpha_{2}}}{\left(t-t_{c}+c\right)^{p}}, t>0$

where $\lambda_{2}(t)$ is, again, the number of aftershocks per day at day $t$ after the main shock, $\alpha_{1}$ and $\alpha_{2}$ depends on the number of aftershocks in sequences 1 and 2, respectively, values of $c$ and $p$ as described in the Omori-Utsu law, and $t_{c}$ is the change point or occurrence time of the large aftershock that triggers a secondary aftershock sequence. Note that both the $c$ and $p$ values in the Omori-Utsu model are related to the heterogeneity of the aftershock zone. Moreover, the two sequences of aftershocks under study mainly occur in the same study region during a short time after the main shock. Therefore, in addition to the model parsimony, it would be reasonable to set the same $c$ and $p$ values in the two sequences of aftershocks. The number of $M>m$ aftershock then obtained as

$$
\lambda_{2}(t, m)=\lambda_{2}(t) S(m)
$$

where $\lambda_{2}(t)$ is given in Eq. (8) and $S(m)$ is, again, as stated in Eq. (2). In fact, Eq. (9) is a combination of the generalized Omori-Utsu model and Gutenberg-Richter law. Therefore, the model in Eq. (9) can be regarded as a generalization of the RJ model to double aftershock sequences and hence denoted by DSRJ model.

When observing the aftershocks with magnitude $m_{i}$ occurring at time $t_{i}, i=1,2, \ldots, n$, after the main shock, the likelihood function of parameter $\theta=\left(\alpha_{1}, \alpha_{2}, c, p, \beta\right)$ in the DSRJ model is given by

$$
L(\theta)=\prod_{i=1}^{n} \lambda_{2}\left(t_{i}\right) \exp \left[-\int_{t_{i-1}}^{t_{i}} \lambda_{2}(s) d s\right] \prod_{i=1}^{n} \beta \exp \left[-\beta\left(m_{i}-M_{c}\right)\right](10)
$$

The associated log-likelihood function is then obtained as

$\ln L(\theta)=\sum_{i=1}^{n} \ln \lambda_{2}\left(t_{i}\right)-\int_{0}^{t_{n}} \lambda_{2}(s) d s+n \ln \beta-\beta \sum_{i=1}^{n}\left(m_{i}-M_{c}\right)$

where

$$
\begin{aligned}
& \int_{0}^{t_{n}} \lambda_{2}(s) d s \\
& =\left\{\begin{array}{cc}
e^{\alpha_{1}} A\left(0, t_{n}, c, p\right) & \text { for } t_{n} \leq t_{c} \\
e^{\alpha_{1}} A\left(0, t_{n}, c, p\right)+e^{\alpha_{2}} A\left(0, t_{n}-t_{c}, c, p\right) & \text { for } t_{n}>t_{c}
\end{array}\right.
\end{aligned}
$$

$A\left(0, t_{n}, c, p\right)$ and $A\left(0, t_{n}-t_{c}, c, p\right)$ can be calculated based on $A\left(T_{1}, T_{2}, c, p\right)$ in Eq. (5) with $T_{1}=0$ and $T_{2}=t_{n}$ and $t_{n}-t_{c}$, respectively. The MLE of $\theta$, denoted by $\hat{\theta}=\left(\hat{\alpha}_{1}, \hat{\alpha}_{2}, \hat{c}, \hat{p}, \hat{\beta}\right)$, is the value of $\theta$ such that $\ln L(\theta)$ in Eq. (11) reaches its maximum. By replacing the $\theta$ with $\hat{\theta}$ in Eq. (9), the MLE of $\lambda_{2}(t, m)$ is then obtained as $\hat{\lambda}_{2}(t, m)=\hat{\lambda}_{2}(t) \hat{S}(m)$. Therefore, the number of $M>m$ aftershocks occurring in the time range $\left(T_{1}, T_{2}\right), 0<T_{1}<T_{2}$, can be estimated or forecasted as

$$
\begin{aligned}
& \hat{N}_{2}(m)=\hat{S}(m) \int_{T_{1}}^{T_{2}} \hat{\lambda}_{2}(t) d t \\
& =\hat{S}(m)\left[e^{\hat{\alpha}_{1}} A\left(T_{1}, T_{2}, \hat{c}, \hat{p}\right)+e^{\hat{\alpha}_{2}} A\left(T_{1}-t_{c}, T_{2}-t_{c}, \hat{c}, \hat{p}\right)\right] \\
& \text { for } T_{1}>t_{c}
\end{aligned}
$$

where $A\left(T_{1}, T_{2}, \hat{c}, \hat{p}\right)$ can be directly calculated based on Eq. (5) and $A\left(T_{1}-t_{c}, T_{2}-t_{c}, \hat{c}, \hat{p}\right)$ can also be computed based on Eq. (5) but with a time shift of $t_{c}$.

Note that all the parameters in the DSRJ model can be estimated based on available data, but the possible change point $t_{c}$ needs to be predetermined. To do so, we may check if there is any large aftershock that triggers a secondary aftershock sequence. Moreover, the Bayesian Information Criterion or BIC (Schwarz 1978) can be used to choose the change point for the DSRJ model. Let $L(\hat{\theta})$ be the estimated likelihood function of $v$ parameters based on $n$ events. Then,

$\mathrm{BIC}=-2 \log L(\hat{\theta})+v \log n$

where $-2 \log L(\hat{\theta})$ measures the goodness-of-fit of the model with $v$ parameters into the data of $n$ events and $v \log n$ represents the penalty of model complexity. Hence, the model with a small BIC is preferred. For example, if $t_{c 1}, t_{c 2}, \ldots, t_{c k}$ are possible $k$ change points in the DSRJ model and the associated BIC values are obtained as $\operatorname{BIC}\left(t_{c 1}\right), \operatorname{BIC}\left(t_{c 2}\right), \ldots$, $\operatorname{BIC}\left(t_{c k}\right)$. We then select the change point that minimizes the associated BIC values, namely, the $\operatorname{argmin}\left\{\operatorname{BIC}\left(t_{c 1}\right)\right.$, $\left.\operatorname{BIC}\left(t_{c 2}\right), \ldots, \operatorname{BIC}\left(t_{c k}\right)\right\}$.

Remark: If there are two possible change points, the RJ model can be generalized to be a triple-sequence RJ model, denoted by TSRJ model. In the TSRJ model, the generalized Omori-Utsu law becomes

$$
\lambda_{3}(t)=\frac{e^{\alpha_{1}}}{(t+c)^{p}}+\frac{e^{\alpha_{2}}}{\left(t-t_{c_{1}}+c\right)^{p}}+\frac{e^{\alpha_{3}}}{\left(t-t_{c_{2}}+c\right)^{p}}, t>0
$$

where $\lambda_{3}(t)$ is the number of aftershocks per day at day $t$ after the main shock, $\alpha_{1}, \alpha_{2}$, and $\alpha_{3}$ depend on the numbers of aftershocks in sequences 1,2 and 3, respectively, values of $c$ and $p$ are as described in the original Omori-Utsu law, $t_{c_{1}}$ and $t_{c_{2}}$ are change points or occurrence times of the large aftershocks that trigger secondary and tertiary aftershock 
sequences, respectively. Parameters in Eq. (14) can be estimated by using the maximum likelihood method and the number of $M>m$ aftershocks occurring in the time range $\left(T_{1}, T_{2}\right), 0<T_{1}<T_{2}$, can be estimated accordingly.

\section{RESULTS}

The $\mathrm{M}_{\mathrm{L}} 6.2$ earthquake with epicentre $\left(24.10^{\circ} \mathrm{N}\right.$, $121.73^{\circ} \mathrm{E}$ ) and hypocentre at depth $10.6 \mathrm{~km}$ occurred at 2350 LT, 6 February 2018 near Hualien, Taiwan (Chen et al. 2019). To have a reliable analysis of the aftershock hazard after the Hualien shock, we employ the earthquake catalogue published by the Central Weather Bureau (CWB) at http://gdms.cwb.gov.tw/index.php. According to the results in Kagan (2002), the possible expanding area of earthquakes after the Hualien shock is about $25\left[=0.02 \times 10^{0.5(6.2)}\right]$ $\mathrm{km}$. To have a more extensive study for the aftershock hazard, particularly, in the golden window for emergent rescue work, however, we consider herein the aftershocks occurred within 3 days after the Hualien shock and in the area within a radius of $30 \mathrm{~km}$ from the main shock epicentre (Fig. 1). The associated magnitude-time plot for the earthquakes is then given in Fig. 2. Note that both the cumulative number of $M>m$ earthquakes (Fig. 3) and the goodness-of-fit measure (Wiemer and Wyss 2000) suggest that the cut-off magnitude for a complete earthquake catalogue is $M_{c}=3.0$. Therefore, under study in this paper are the $M \geq 3.0$ aftershocks occurred within 3 days after the Hualien shock and within the dashed circle in Fig. 1.
Based on the aftershocks under study, we compute the $b$-values sequentially for each set of 50 events (Fig. 4). We also calculate the occurrence rate per day based on consecutive 10 events and estimate the Omori-Utsu law based on all the $M \geq 3.0$ aftershocks under study (Fig. 5). An investigation of the results in Figs. 4 and 5, simultaneously, reveals that the time-varying $b$-values have two dropdowns in the aftershock sequence at which the occurrence rates of aftershocks have unusual risings. These observations indicate that two possible large aftershocks may change the aftershock hazard where one is the $\mathrm{M}_{\mathrm{L}}=5.4$ aftershock occurred on $0315 \mathrm{LT}, 7$ February 2018 at $\left(24.01^{\circ} \mathrm{N}, 121.73^{\circ} \mathrm{E}\right)$ and the other is the $\mathrm{M}_{\mathrm{L}}=5.8$ aftershock occurred on $2321 \mathrm{LT}$, 7 February 2018 at $\left(24.08^{\circ} \mathrm{N}, 121.78^{\circ} \mathrm{E}\right)$, and the two aftershocks are all shallow at depths of 5.6 and $7.8 \mathrm{~km}$, respectively. Since both the aftershocks bring up an increase in the occurrence rate of $M \geq 3.0$ aftershocks, we employ the DSRJ model in Eq. (9) with possible change point at local time 0315 or 2321, 7 February 2018 to describe the aftershock hazard. Note that the associated DSRJ models are denoted by DSRJ(3) and DSRJ(24), respectively, since the two possible change points are about 3 and $24 \mathrm{hrs}$ after the main shock. After choosing the change point and hence the model with BIC values, the occurrence rate of forthcoming aftershocks within three days after the Hualien shock is forecasted.

Let $T$ be the time (in day) from the occurrence of main shock at which the hazard of $M \geq 3.0$ or $M \geq 4.0$ aftershocks is under forecast. To do so, the RJ, DSRJ(3) and DSRJ(24)

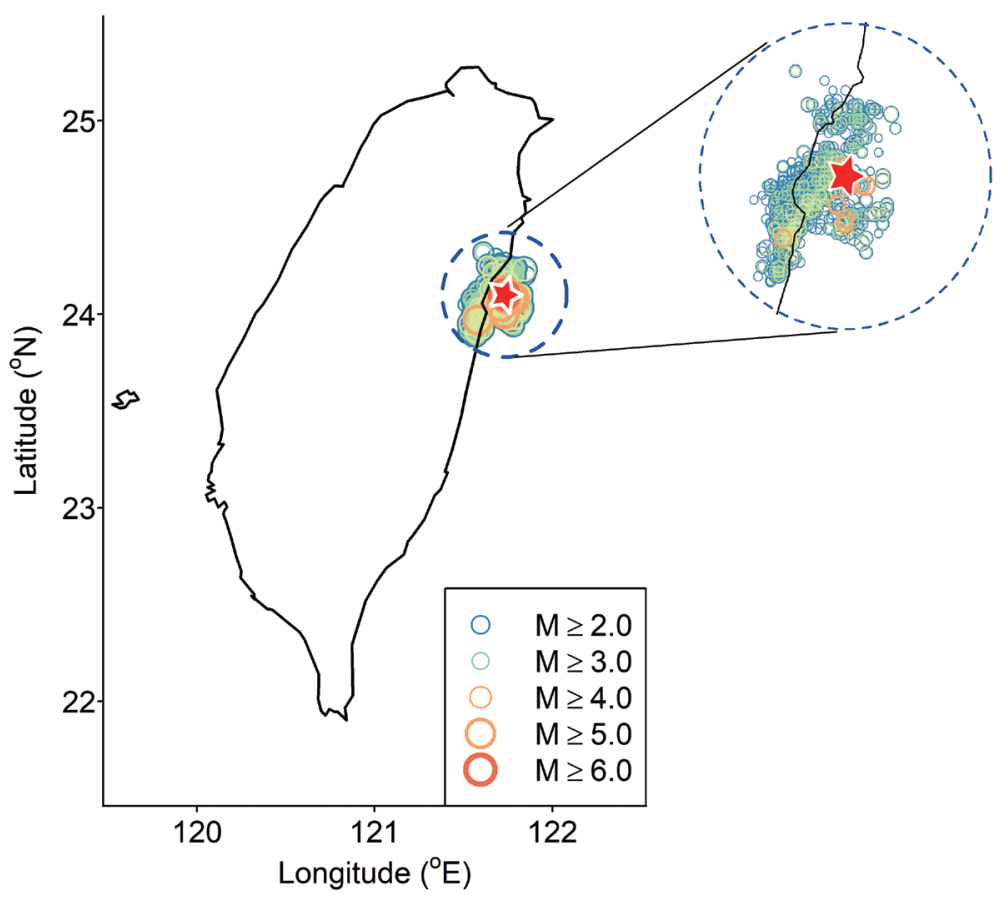

Fig. 1. Earthquakes occurred within 3 days after the 2018 February $6 \mathrm{M}_{\mathrm{L}} 6.2$ Hualien shock. The red star locates the epicenter of the Hualien shock at $\left(24.10^{\circ} \mathrm{N}, 121.73^{\circ} \mathrm{E}\right)$ and earthquakes under study are in the dashed circle with radius of $32 \mathrm{~km}$ centred at the epicenter. 


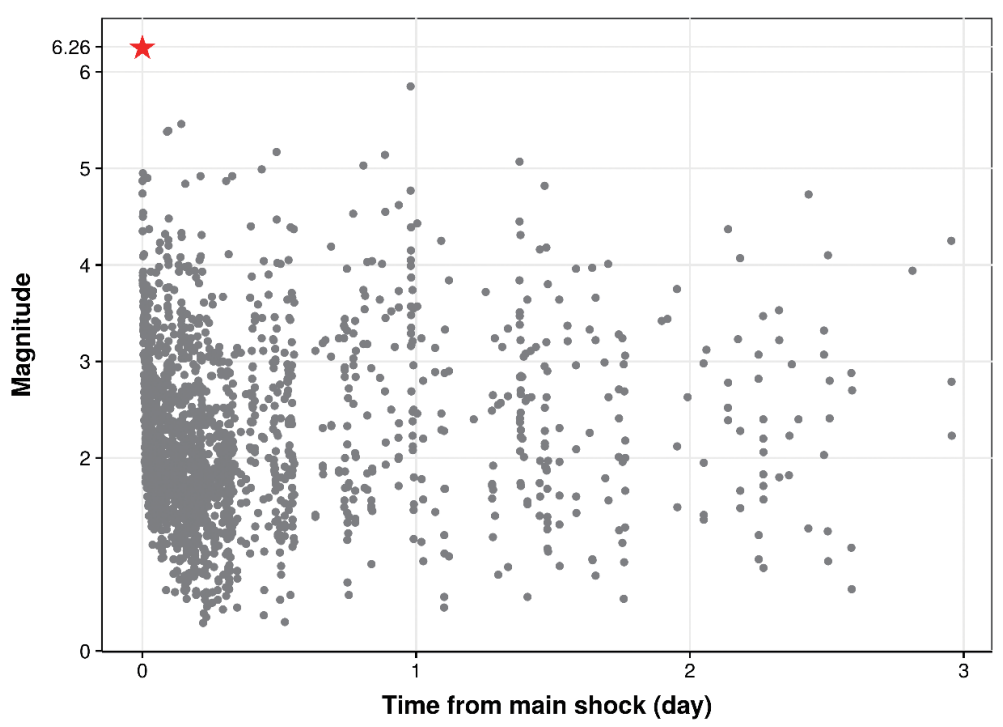

Fig. 2. The magnitude of earthquakes after the Hualien shock. The red star is the magnitude of the main shock.

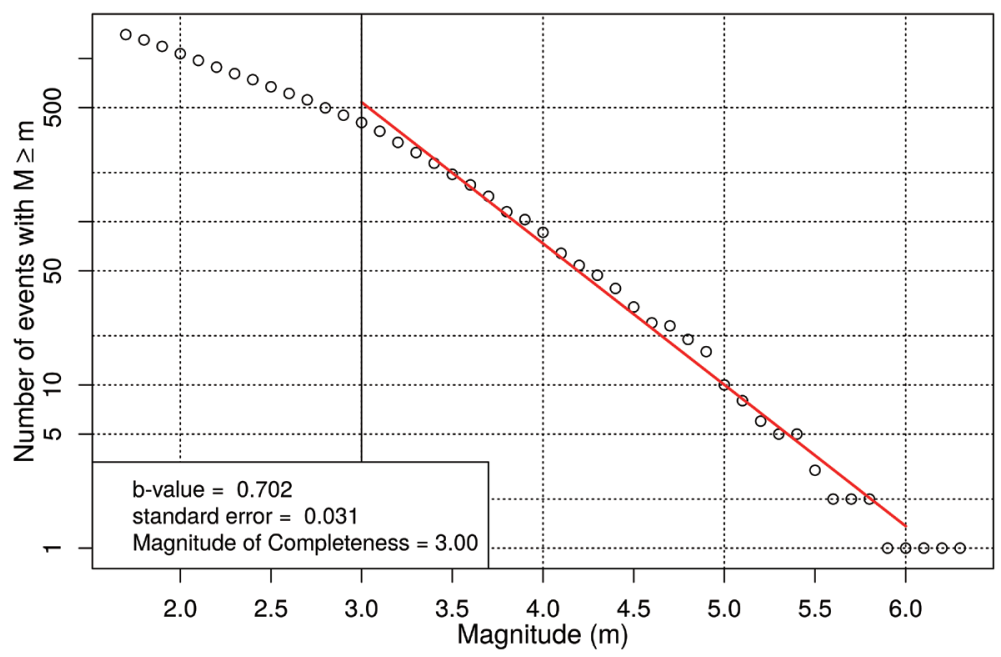

Fig. 3. Cumulative numbers of $M \geq m$ earthquakes as functions of $m$ for earthquakes occurred within 3 days after the Hualien shock in the study region. The red line is the estimated Gutenberg-Richter law.

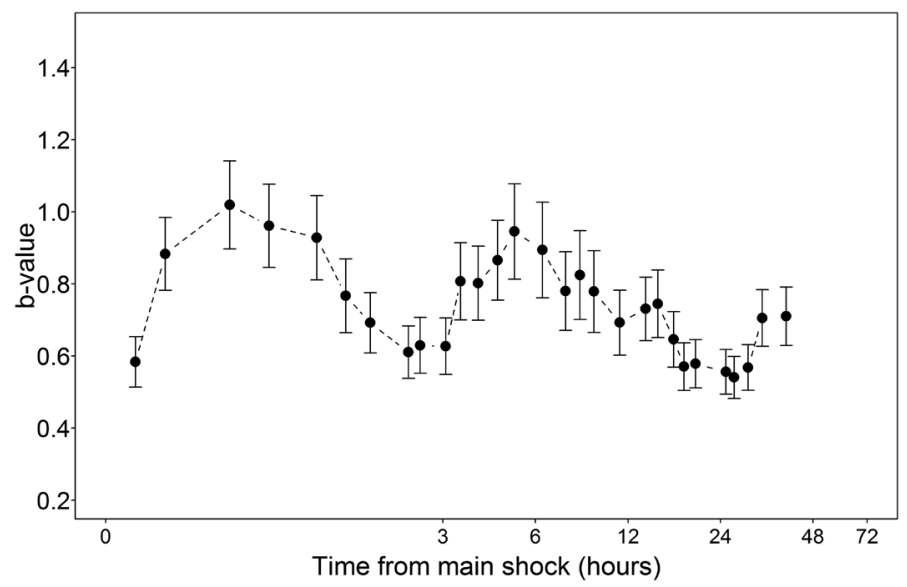

Fig. 4. The time-varing $b$-values and the associated standard error for $M \geq 3.0$ earthquakes after the Hualien shock. 


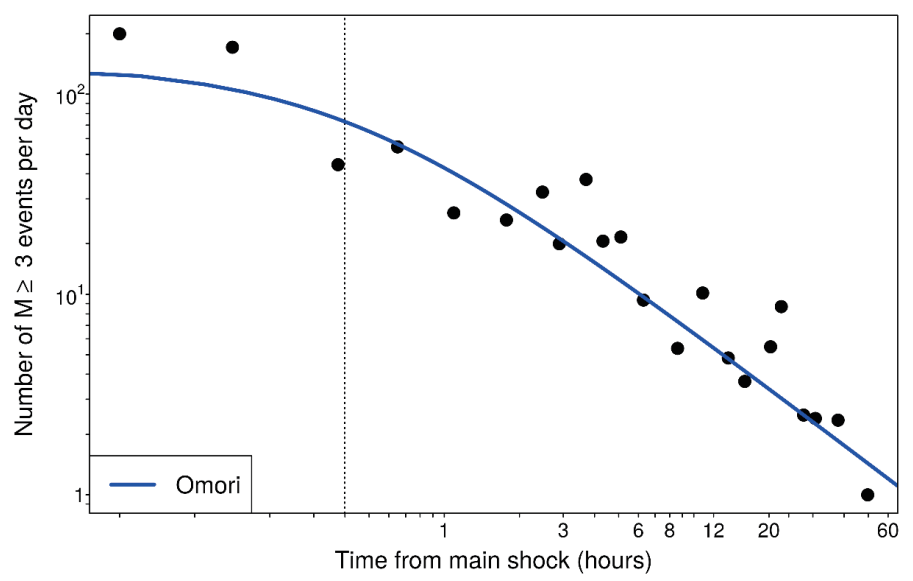

Fig. 5. The occurrence rate of $M \geq 3.0$ earthquakes after the Hualien shock. Black dots are the observed occurrence rates and the blue line is the estimated Omori-Utsu law.

models are respectively estimated based on $M \geq 3.0$ earthquakes occurred within time $T$ after the main shock. The BIC values, as shown in Table 1, suggest that the DSRJ(3) model is more appropriate for describing the available data than the RJ or DSRJ(24) model. Therefore, we denote the DSRJ(3) by DSRJ, hereafter, and the estimated parameters of RJ and DSRJ models are presented in Table 2, respectively. The estimated RJ and DSRJ models are then used to forecast the occurrence rate of $M \geq 3.0$ or $M \geq 4.0$ aftershocks at any time from $T$ to 3 days after the main shock (Figs. 6 and 7). The numbers of forthcoming $M \geq 3.0$ or $M$ $\geq 4.0$ aftershocks in the future 3-T days or 72-24T hrs are further forecasted based on the estimated DSRJ or RJ model (Fig. 8). The time $T$ under study is $6,12,24,30,36$ or 48 hrs after the main shock as shown in Fig. 8, and the results for evaluating aftershock hazard at $T=6,12,24$, and $36 \mathrm{hrs}$ after the main shock are presented in Figs. 6 and 7.

\section{DISCUSSION AND CONCLUSION}

The double-sequence model or DSRJ model is constructed to describe the hazard of aftershock of Hualien event. The results of data analysis confirm that the DSRJ model with a change point at 0315 LT, 7 February 2018, where an aftershock of $\mathrm{M}_{\mathrm{L}}=5.4$ may change the seismicity rate and the magnitude distribution, outperforms the widely used single-sequence RJ model (Reasenberg and Jones $1989,1994)$ on near real-time assessment of aftershock hazard. In fact, we not only present the results of a retrospective study for the Hualien main shock, but also suggest the Bayesian Information Criterion (BIC) to select an optimal model or determine an appropriate change point in the DSRJ model for a better forecast of occurrence rates or number of forthcoming aftershocks. Therefore, the DSRJ model along with the statistical methods would be helpful for aftershock hazard assessment in any situation where appeared with a secondary aftershock sequence.

As a matter of fact, the double-sequence aftershock hazard model with one change point can be extended to a multiple-sequence aftershock hazard model with more than one change point if there are several aftershock sequences possibly triggered by large aftershocks. For example, after the Hualien main shock, there are two possible change points at 0315 and 2321 LT, respectively, 7 February 2018. Taking both the change points into account to build the triple-sequence model, denoted by TSRJ, we estimate the TSRJ model based on $M \geq 3.0$ aftershocks after the main shock. The estimated TSRJ models then give BIC values as -1031.7 and -1023.5 based on data available at 24 and $36 \mathrm{hrs}$, respectively. Note that the BIC values, comparing with those in Table 1, do not support the TSRJ model for describing the aftershock hazard. Moreover, the results in Fig. 9 demonstrate that the TSRJ may not give more accurate forecast of the occurrence rates of future $M \geq 3.0$ aftershocks than the DSRJ model, especially at $24 \mathrm{hrs}$ or one day after the main shock. Therefore, multiple-sequence aftershock hazard models may not perform better than the proposed DSRJ model on assessing the aftershock hazard of the Hualien main shock.

Note that a $\mathrm{M}_{\mathrm{L}} 5.8$ earthquake occurred at $2156 \mathrm{LT}$, 4 February 2018, triggered an aftershock sequence in the study region (Fig. 1). To describe the hazard of earthquakes after the $\mathrm{M}_{\mathrm{L}} 5.8$ earthquake, we consider a double-sequence model with a change point at the occurrence time of the $\mathrm{M}_{\mathrm{L}}$ 6.2 main shock, denoted by FDSRJ. If two change points at local time 2350 on 6 February and 0315 on 7 February 2018 , are both under study, then it leads to a triple-sequence model, denoted by FTSRJ. The results in Fig. 10 show that the FDSRJ and FTSRJ are competitive to the DSRJ when forecasting the occurrence rates of $M \geq 3.0$ aftershocks at $6 \mathrm{hrs}$ after the $\mathrm{M}_{\mathrm{L}} 6.2$ main shock. However, the FDSRJ or FTSRJ may not perform better than the proposed DSRJ 
Table 1. The BIC value of the estimated model based on $M \geq 3.0$ aftershocks after the Hualien shock.

\begin{tabular}{c|cccccc}
\hline \multirow{2}{*}{ Model } & \multicolumn{6}{|c}{ Time form main shock (hrs) } \\
\cline { 2 - 7 } & $\mathbf{6}$ & $\mathbf{1 2}$ & $\mathbf{2 4}$ & $\mathbf{3 0}$ & $\mathbf{3 6}$ & $\mathbf{4 8}$ \\
\hline RJ & -871.6 & -937.2 & -1020.5 & -994.0 & -995.6 & -974.8 \\
DSRJ(3) & -889.1 & -956.6 & -1036.8 & -1031.9 & -1025.3 & -998.6 \\
DSRJ(24) & - & - & -1030.2 & -1028.9 & -1020.1 & -995.4 \\
\hline
\end{tabular}

Table 2. Estimated model parameters based on $M \geq 3.0$ earthquakes after the Hualien shock.

\begin{tabular}{c|cccc|cccccc}
\hline \multirow{2}{*}{$\begin{array}{c}\text { Time (hr) } \\
\text { form main shock }\end{array}$} & \multicolumn{4}{|c|}{ RJ model } & \multicolumn{5}{c}{ DSRJ model } \\
\cline { 2 - 10 } & $\boldsymbol{\alpha}$ & $\boldsymbol{c}$ & $\boldsymbol{p}$ & $\boldsymbol{\beta}$ & $\boldsymbol{\alpha}_{1}$ & $\boldsymbol{\alpha}_{\mathbf{2}}$ & $\boldsymbol{c}$ & $\boldsymbol{p}$ & $\boldsymbol{\beta}$ \\
\hline 6 & 3.647 & 0.034 & 0.539 & 1.690 & 3.674 & 2.446 & 0.160 & 0.874 & 1.690 \\
12 & 3.584 & 0.040 & 0.638 & 1.695 & 3.468 & 2.113 & 0.057 & 0.786 & 1.695 \\
24 & 3.614 & 0.056 & 0.664 & 1.615 & 3.449 & 2.014 & 0.039 & 0.720 & 1.615 \\
30 & 3.683 & 0.101 & 0.742 & 1.611 & 3.449 & 2.031 & 0.042 & 0.739 & 1.611 \\
36 & 3.260 & 0.005 & 0.612 & 1.600 & 3.461 & 2.069 & 0.050 & 0.757 & 1.600 \\
48 & 3.766 & 0.153 & 0.797 & 1.614 & 3.474 & 2.134 & 0.062 & 0.801 & 1.614 \\
\hline
\end{tabular}

Note: The DSRJ model is the one with change point at 0315 LT, 7 February 2018.
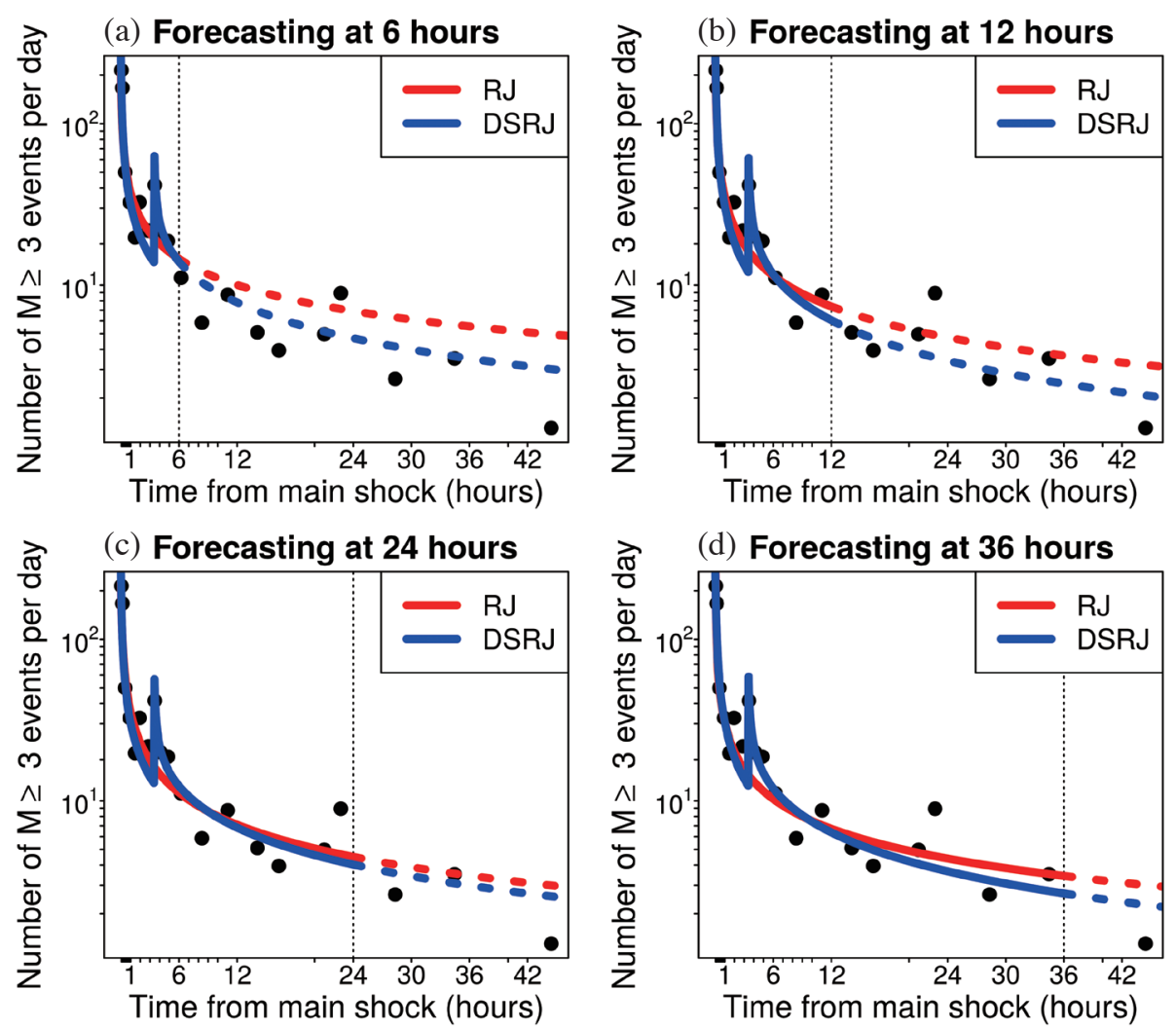

Fig. 6. The occurrence rate of $M \geq 3.0$ earthquakes after the Hualien shock. Solid and dashed lines represent the estimated and forecasted occurrence rates, respectively, based on the RJ and DSRJ models, black dots are the observed occurrence rates, and the vertical dotted line indicates the forecasting time. 

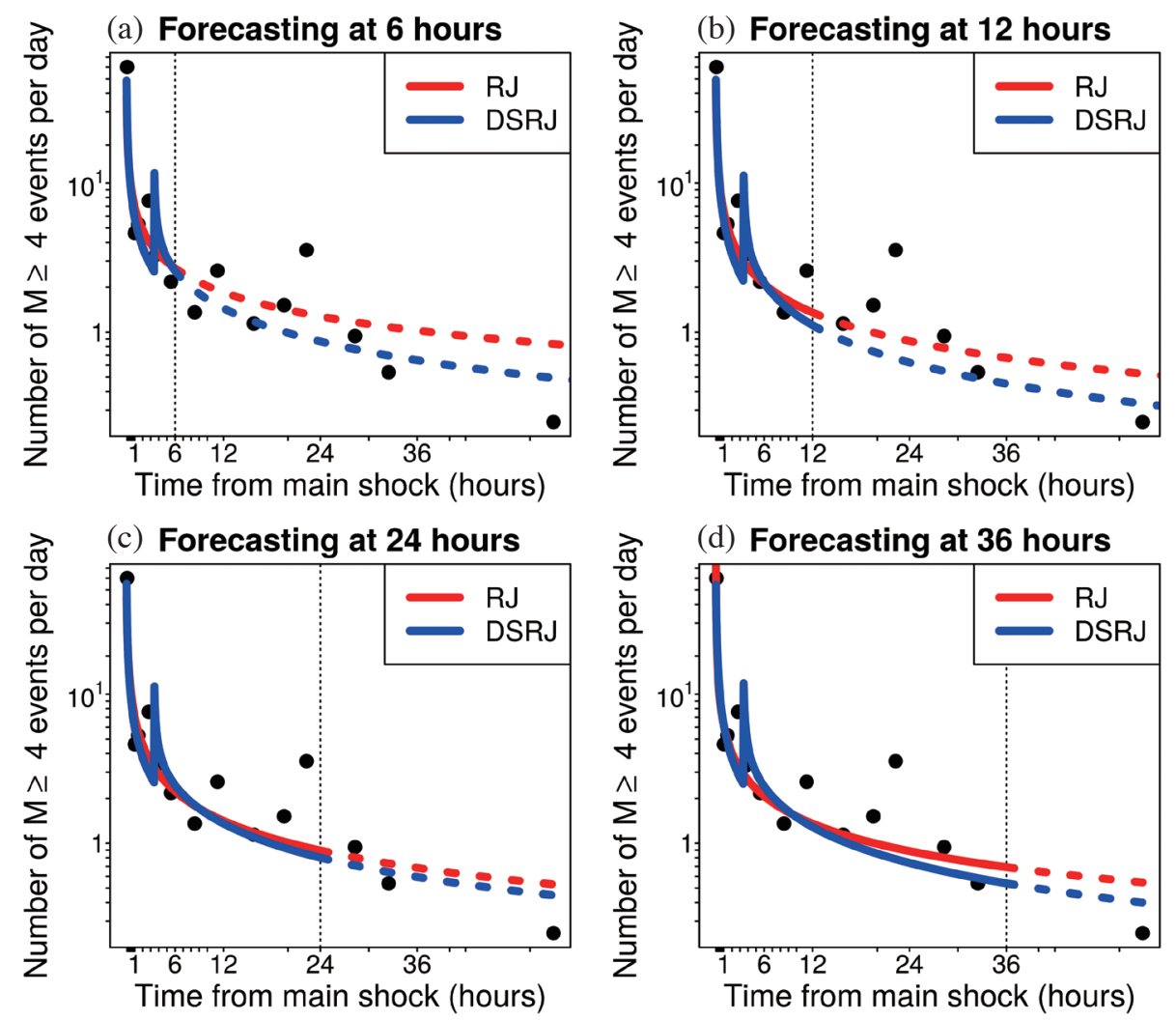

Fig. 7. The occurrence rate of $M \geq 4.0$ earthquakes after the Hualien shock. Solid and dashed lines represent the estimated and forecasted occurrence rates, respectively, based on the RJ and DSRJ models, black dots are the observed occurrence rates, and the vertical dotted line indicates the forecasting time.
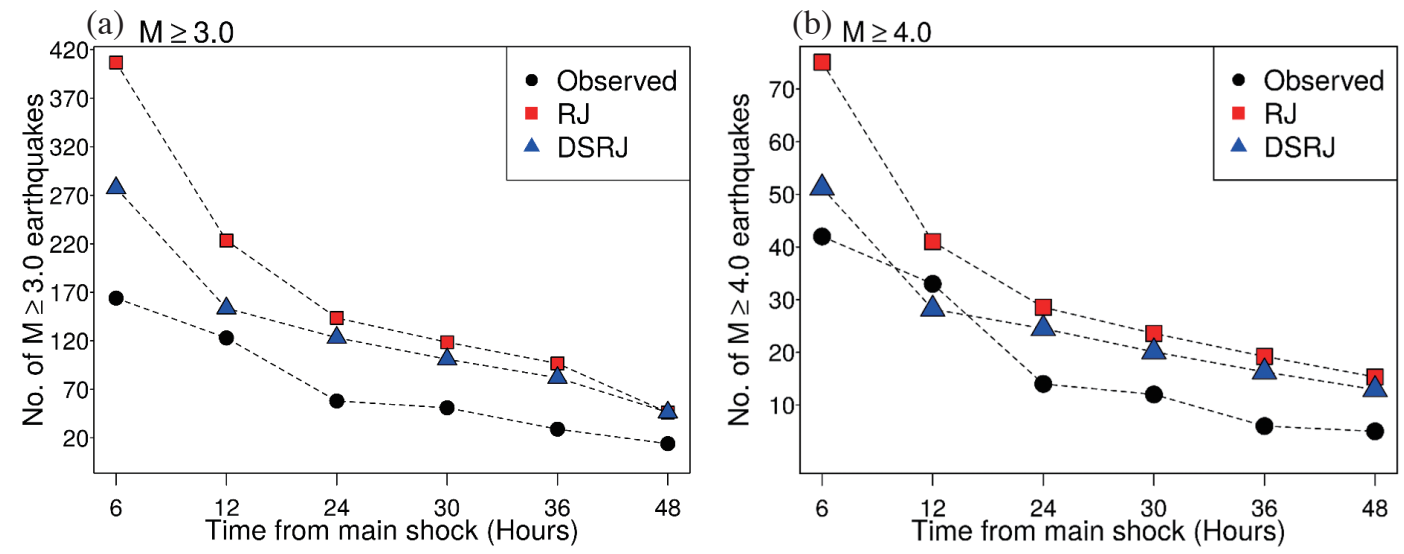

Fig. 8. Number of forthcoming $M \geq 3.0$ or $M \geq 4.0$ earthquakes within 3 days after the Hualien shock. 

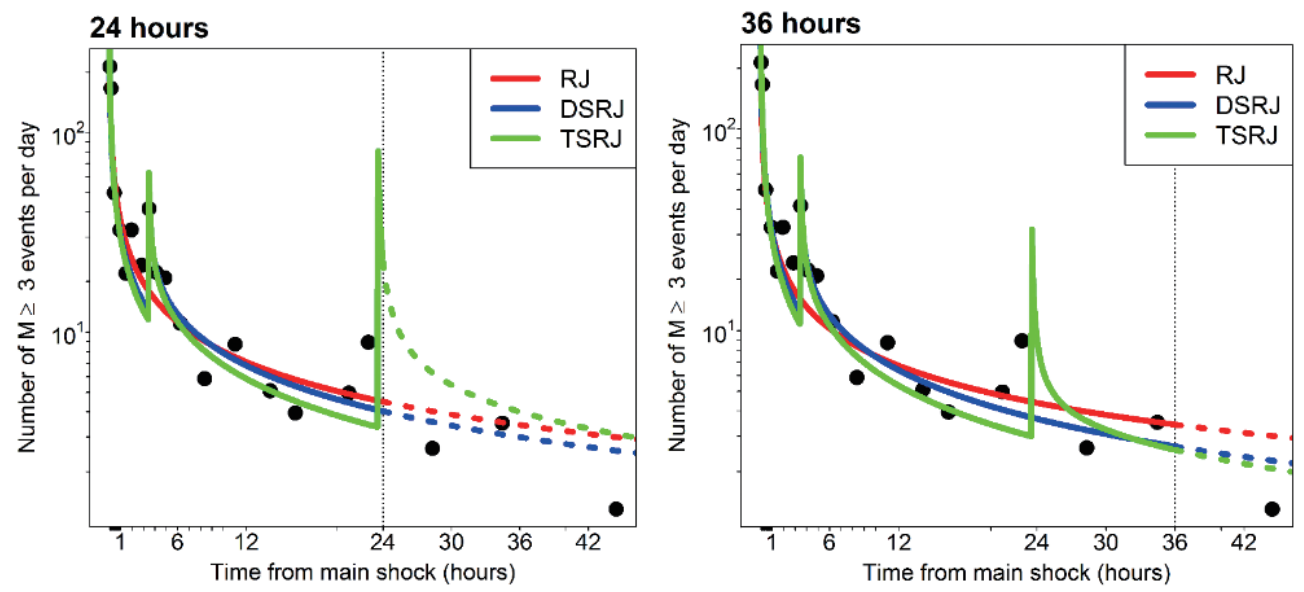

Fig. 9. The occurrence rate of $M \geq 3.0$ earthquakes after the Hualien shock. Solid and dashed lines represent the estimated and forecasted occurrence rates, respectively, based on the RJ, DSRJ, and TSRJ models, black dots are the observed occurrence rates, and the vertical dotted line indicates the forecasting time.
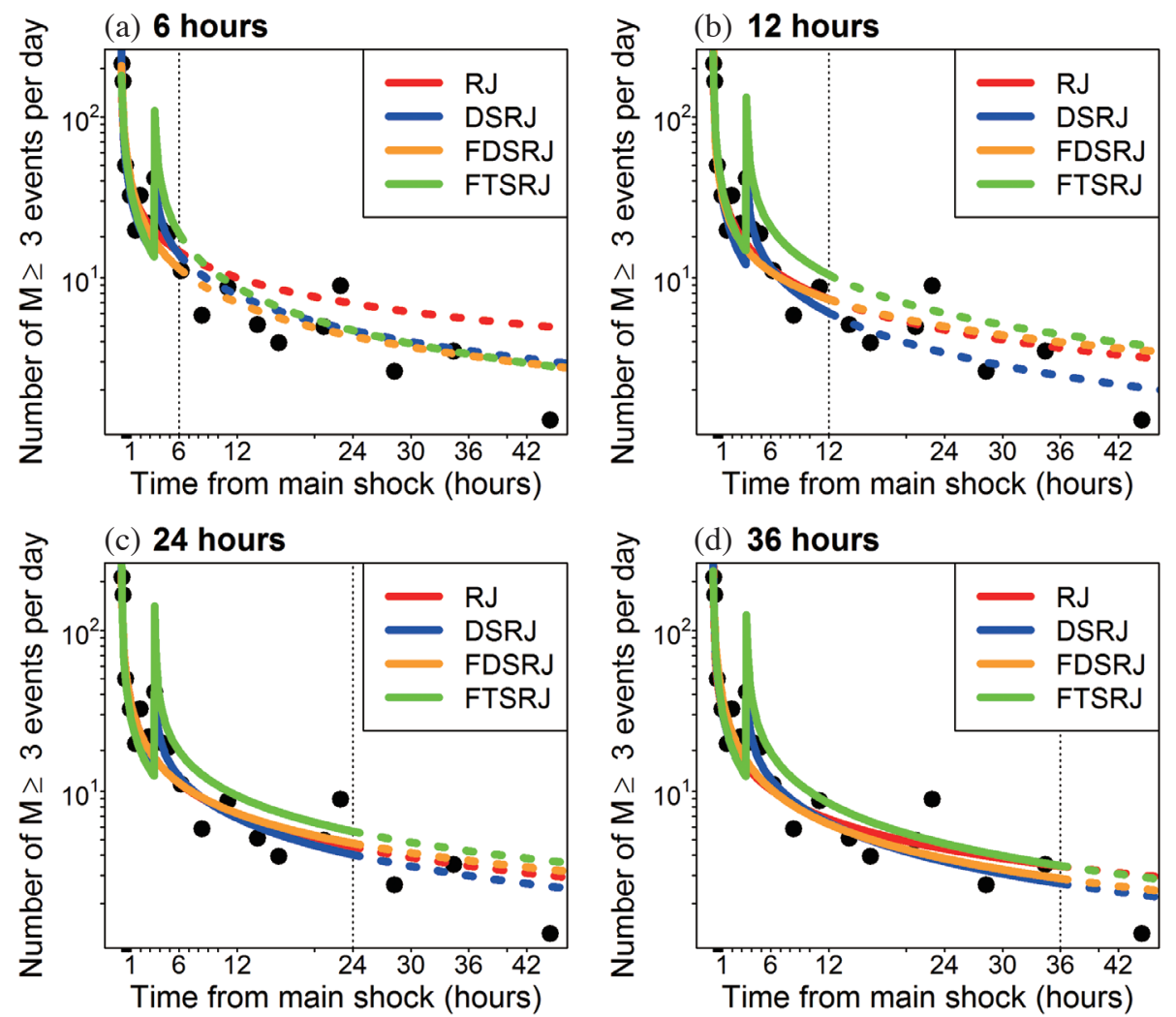

Fig. 10. The occurrence rate of $M \geq 3.0$ earthquakes after the Hualien shock. Solid and dashed lines represent the estimated and forecasted occurrence rates, respectively, based on the RJ, DSRJ, FDSRJ, and FTSRJ models, black dots are the observed occurrence rates, and the vertical dotted line indicates the forecasting time. 
model when forecasting at $12 \mathrm{hrs}$ or later after the main shock. These observations indicate that incorporating the aftershock sequence about 2 days before the $M_{L} 6.2$ main shock into the DSRJ or TSRJ model may not help the assessment of aftershock hazard after the main shock. Therefore, how to effectively use previous earthquake sequences to evaluate the current aftershock hazard would be an important problem for further study.

When previous earthquakes in the study area are incorporated into the current aftershocks to estimate the Gutenberg-Richter law (Gutenberg and Richter 1944), the resulted $b$-value would be larger than that computed from only the current aftershocks. For example, the $b$-values are $0.74,0.74,0.70,0.70,0.70$, and 0.70 based on $M \geq 3.0$ aftershocks available at $6,12,24,30,36$, and $48 \mathrm{hrs}$ after the Hualien shock. However, the $b$-values become $0.76,0.76$, $0.74,0.74,0.74$, and 0.74 when taking additionally the $M \geq$ 3.0 earthquakes occurred from 2017/1/1 till 2350 LT, 6 February 2018. Hence, the forecasted number of forthcoming $M \geq 4.0$ aftershocks based on either the RJ or DSRJ model becomes smaller than the one shown in Fig. 8b. However, though the results are not shown herein, the DSRJ model still gives a better forecast of the number of forthcoming $M$ $\geq 4.0$ aftershocks than the RJ model when forecasting at 6 hrs after the Hualien main shock.

Finally, the DSRJ model is not an over-simplified ETAS model (Ogata 1988). In the ETAS model, any aftershocks are triggered by all the previous aftershocks and hence the computation of parameters is very time-consuming, although there are only three parameters. On the contrary, the DSRJ model have four parameters and is built by choosing a change point at which one large aftershock has the greatest influence on the occurrence of later ones. Therefore, an application of the DSRJ model and the BIC gives a more flexible model for the data and produces more quickly the forecasted results for assessing the aftershock hazard. The advantage on using the DSRJ model is time management, which is of great importance, particularly when the information of aftershock hazard is of urgent need for emergent rescue in the golden window after the large main shock.

Acknowledgements This study is supported by the Taiwan Ministry of Science and Technology (MOST) grant MOST 106-2628-M-008-002. The authors would like to thank the reviewers for constructive comments and suggestions which lead to an improvement on the presentation of the materials.

\section{REFERENCES}

Aki, K., 1965: Maximum likelihood estimate of $\mathrm{b}$ in the formula $\log \mathrm{N}=\mathrm{a}-\mathrm{bM}$ and its confidence limits. Bull. Earthq. Res. Inst., Tokyo Univ., 43, 237-239.

Chen, P.-F., Y.-L. Chen, P.-L. Su, Y.-D. Peng, and L.-F.
Chen, 2019: Understanding the 6 February 2018, Hualien earthquake sequence through catalog compilation. Terr. Atmos. Ocean. Sci., 30, 399-409, doi: 10.3319/ TAO.2018.11.15.02. [Link]

Gerstenberger, M. C., S. Wiemer, L. M. Jones, and P. A. Reasenberg, 2005: Real-time forecasts of tomorrow's earthquakes in California. Nature, 435, 328-331, doi: 10.1038/nature03622. [Link]

Gerstenberger, M. C., L. M. Jones, and S. Wiemer, 2007: Short-term aftershock probabilities: Case studies in California. Seismol.Res. Lett., 78, 66-77, doi: 10.1785/ gssrl.78.1.66. [Link]

Gutenberg, R. and C. F. Richter, 1944: Frequency of earthquakes in California. Bull. Seismol. Soc. Am., 34, 185188.

Kagan, Y. Y., 2002: Seismic moment distribution revisited: I. Statistical results. Geophys. J. Int., 148, 520-541, doi: 10.1046/j.1365-246x.2002.01594.x. [Link]

Kisslinger, C. and L. M. Jones, 1991: Properties of aftershock sequences in southern California. J. Geophys. Res., 96, 11947-11958, doi: 10.1029/91JB01200. [Link]

Nanjo, K. Z., B. Enescu, R. Shcherbakov, D. L. Turcotte, T. Iwata, and Y. Ogata, 2007: Decay of aftershock activity for Japanese earthquakes. J. Geophys. Res., 112, 1B08309, doi: 10.1029/2006JB004754. [Link]

Narteau, C., S. Byrdina, P. Shebalin, and D. Schorlemmer, 2009: Common dependence on stress for the two fundamental laws of statistical seismology. Nature, $\mathbf{4 6 2}$, 642-645, doi: 10.1038/nature08553. [Link]

Ogata, Y., 1983: Estimation of the parameters in the modified Omori formula for aftershock frequencies by the maximum likelihood procedure. J. Phys. Earth, 31, 115-124, doi: 10.4294/jpe1952.31.115. [Link]

Ogata, Y., 1988: Statistical models for earthquake occurrences and residual analysis for point processes. $J$. Am. Stat. Assoc., 83, 9-27, doi: 10.2307/2288914. [Link]

Omi, T., Y. Ogata, Y. Hirata, and K. Aihara, 2013: Forecasting large aftershocks within one day after the main shock. Sci.Rep., 3, doi: 10.1038/srep02218. [Link]

Omori, F., 1984: On the aftershocks of earthquake. J. ColI. Sci. Imp. Univ. Tokyo, 7, 111-200.

Reasenberg, P. A. and L. M. Jones, 1989: Earthquake hazard after a mainshock in California. Science, 243, 11731176, doi: 10.1126/science.243.4895.1173. [Link]

Reasenberg, P. A. and L. M. Jones, 1994: Earthquake aftershocks: Update. Science, 265, 1251-1252, doi: 10.1126/science.265.5176.1251. [Link]

Schwarz, G., 1978: Estimating the dimension of a model. Ann.Statist., 6,461-464, doi: 10.1214/aos/1176344136. [Link]

Utsu, T., 1961: A statistical study on the occurrence of aftershocks. Geophys. Mag., 30, 521-605. 
Utsu, T., Y. Ogata, and R. S. Matsu'ura, 1995: The centenary of the Omori formula for a decay law of aftershock activity. J. Phys. Earth, 43, 1-33, doi: 10.4294/ jpe1952.43.1. [Link]

Wiemer, S. and K. Katsumata, 1999: Spatial variability of seismicity parameters in aftershock zones. J. Geophys. Res., 104, 13135-13151, doi: 10.1029/1999JB900032. [Link]

Wiemer, S. and M. Wyss, 2000: Minimum magnitude of completeness in earthquake catalogs: Examples from Alaska, the western United States, and Japan. Bull. Seismol. Soc. Am., 90, 859-869, doi: 10.1785/0119990114. [Link]

Wiemer, S., M. Gerstenberger, and E. Hauksson, 2002: Properties of the aftershock sequence of the $1999 M_{\mathrm{w}}$ 7.1 Hector Mine earthquake: Implications for aftershock hazard. Bull. Seismol. Soc. Am., 92, 1227-1240, doi: 10.1785/0120000914. [Link] 